\title{
SPACE-TIME CONTINUA OF PERFECT FLUIDS IN GENERAL RELATIVITY*
}

\author{
BY
}

\author{
LUTHER PFAHLER EISENHART
}

When the expressions proposed by Einstein for the components of the energy-momentum tensor of matter in the state of a perfect fluid are substituted in the field equations of general relativity, these equations impose conditions to be satisfied by the space-time continuum of a perfect fluid. It is the purpose of this paper to give a geometrical characterization of these continua; to determine the conditions that the world-lines of flow be geodesics; to show that there is a geometry of paths for the space of a perfect fluid for which the world-lines of flow and of light are paths and that it is possible to find a space in correspondence with the given space such that the world-lines of flow and of light of the latter are represented by geodesics of the former; to indicate the significance of the cosmological solutions of Einstein and de Sitter in the general theory; and to determine the radially symmetric continua of a static fluid for which the spaces have constant Riemannian curvature.

1. Einstein space of a perfect fluid. Consider a four-dimensional Riemann space with the fundamental quadratic form

$$
d s^{2}=g_{i j} d x^{i} d x^{j} \quad(i, j=1,2,3,4)
$$

which is the space-time continuum of matter. We adopt Einstein's hypothesis that at each point of space (1.1) is reducible to the form

$$
d s^{2}=-\left(d X^{1}\right)^{2}-\left(d X^{2}\right)^{2}-\left(d X^{3}\right)^{2}+\left(d X^{4}\right)^{2},
$$

where the $d X^{i}$ are linear transforms of the $d x^{i}$ with real coefficients. Adopting the terminology of algebra, we say that at each point the signature of (1.2) is -2 , and thus we say that the signature of (1.1) is -2 . As a consequence we have that the determinant of the $g_{i j}$ 's is negative, that is

$$
g \equiv\left|g_{i j}\right|<0
$$

\footnotetext{
* Presented to the Society under a different title, October 27, 1923.
} 
The field equations of relativity are

$$
R_{i j}-\frac{1}{2} g_{i j} R=-k T_{i j},
$$

where $k$ is a constant, $T_{i j}$ is the energy-momentum tensor, $R_{i j}$ is the contracted Riemann tensor formed with respect to (1.1) and

$$
R=R_{i}^{i}=g^{i j} R_{i j},
$$

$g^{i j}$ being the cofactor of $g_{i j}$ in $g$ divided by $g$.

If we put

$$
u^{i}=\frac{d x^{i}}{d s}, \quad u_{i}=g_{i j} u^{j},
$$

the components of $T_{i j}$ for a perfect fluid, as suggested by Einstein, are

$$
T_{i j}=\sigma u_{i} u_{j}-p g_{i j} .
$$

As defined, the $u^{i}$ are the contravariant components of the tangent to the world-lines of flow of the fluid. The scalar $p$ is the hydrostatic pressure, and $\sigma-p$ is the density of matter or energy per unit volume.

2. Ricci's principal directions. If $\varrho_{h}$ is any root of the determinant equation

$$
\left|R_{i j}+\varrho g_{i j}\right|=0,
$$

the functions $\lambda_{h}^{i}$ defined by

$$
\left(R_{i j}+\varrho_{h} g_{i j}\right) \lambda_{h}^{i}=0
$$

are the contravariant components of a Ricci* principal direction of the space. For any Riemann space of $n$ dimensions, when the roots of (2.1) are simple, $n$ principal directions are uniquely determined by (2.2), and any two of these directions at a point are orthogonal. If a root of (2.1) is multiple, say of order $r$, and the elementary divisors are simple, the directions corresponding to this root are linearly expressible in terms of $r$ mutually orthogonal directions, which are orthogonal also to the directions corresponding to any other root. Hence when all the elementary divisors

*Atti del Reale Istituto Veneto, vol. 63 (1904), pp. 1233-39; also, Eisenhart, Proceedings of the National Academy of Sciences, vol. 8 (1922), p. 24. 
are simple, an orthogonal $n$-uple of principal directions can be found, their contravariant components being denoted by $\lambda_{h}^{i}(h, i=1, \ldots, n)$, where $h$ indicates the direction. Equation (2.2) may be replaced by

$$
R_{i j}=-\sum_{h}^{1, \ldots, n} \varrho_{h} \lambda_{h, i} \lambda_{h, j}
$$

where

$$
\lambda_{h, i}=g_{i j} \lambda_{h}^{j}
$$

3. Geometric characterization of the spaces of a perfect fluid. When we substitute in (2.2) the expression for $R_{i j}$ from (1.4) and (1.7), we obtain

$$
\left[k \sigma u_{i} u_{j}-g_{i j}\left(\varrho_{h}+\frac{1}{2} R+k p\right)\right] \lambda_{h}^{i}=0 \text {. }
$$

If we take

$$
\lambda_{1}^{i}=u^{i}
$$

and make use of (1.6) and

$$
g_{i j} u^{i} u^{j}=u^{i} u_{i}=1,
$$

which follows from (1.1) and (1.6), we obtain

$$
\left[k(\sigma-p)-\frac{1}{2} R-\varrho_{1}\right] u_{j}=0 \quad(j=1, \ldots, 4) .
$$

Hence we have

$$
\varrho_{1}=k(\sigma-p)-\frac{1}{2} R .^{*}
$$

If $\lambda_{h}^{i}$ are the contravariant components of any vector orthogonal to $u^{i}$, that is

$$
g_{i j} u^{i} \lambda_{h}^{j}=u_{j} \lambda_{h}^{j}=0 \text {, }
$$

then (2.1) and (2.2) are satisfied by

$$
\varrho_{h}=-\left(\frac{1}{2} R+k p\right)
$$

* For this value of $\rho$ equation (2.1) reduces to $\left|u_{i} u_{j}-g_{i j}\right|=0$, which can readily be shown to be a consequence of (1.6). 
Since every vector orthogonal to $u^{i}$ satisfies this condition, it follows that $\varrho_{h}$ is a triple root of (2.1) and the elementary divisors are simple. Hence we have the theorem:

In the four-dimensional space-time continuum of a perfect fuid in general relativity, one of the roots of (2.1) is simple and there is a triple root with simple elementary divisors; the world-line of flow is the principal direction determined by the simple root.

Consider, conversely, a Riemann space of four dimensions for which at each point the linear element is reducible to (1.2) by a real transformation of the form

$$
d X^{i}=a_{j}^{i} d x^{j} \quad(i, j=1, \ldots, 4) .
$$

Then the quantities $a_{j}^{i}$ must be such that at each point

$$
\left(d X^{4}\right)^{2}-d s^{2}=\left(a_{i}^{4} a_{j}^{4}-g_{i j}\right) d x^{i} d x^{j}
$$

is of rank 3 and signature 3. Suppose further that (2.1) admits a simple root $\varrho^{\prime}$ and a triple root $\varrho^{\prime \prime}$ with simple elementary divisors. If $\lambda_{1}^{i}$ are the components of the vector determined by $\varrho^{\prime}$ and $g_{i j} \lambda_{1}^{i} \lambda_{1}^{j}>0$, then $u^{i}=\lambda_{1}^{i} / \sqrt{g_{i j} \lambda_{1}^{i} \lambda_{1}^{j}}$ are real and satisfy (3.3). In order that $u^{i}$ be the components of a velocity vector, it must be possible to obtain a transformation (3.7) at each point such that in the direction of the vector $d X^{4} \neq 0, d X^{\alpha}=0$ $(\alpha=1,2,3)$. Hence the quantities $a_{j}^{i}$ must satisfy also the conditions

$$
u^{j} a_{j}^{4} \neq 0, \quad u^{j} a_{j}^{\alpha}=0 \quad(\alpha=1,2,3) .
$$

Assuming that these conditions are satisfied, we have from equation (2.3)

$$
\begin{aligned}
R_{i j} & =-\varrho^{\prime} u_{i} u_{j}-\varrho^{\prime \prime} \sum_{h}^{2,8,4} \lambda_{h, i} \lambda_{h, j} \\
& =\left(\varrho^{\prime \prime}-\varrho^{\prime}\right) u_{i} u_{j}-\varrho^{\prime \prime}\left(u_{i} u_{j}+\sum_{h}^{2,3,4} \lambda_{h, i} \lambda_{h, j}\right) \\
& =\left(\varrho^{\prime \prime}-\varrho^{\prime}\right) u_{i} u_{j}-\varrho^{\prime \prime} g_{i j} .
\end{aligned}
$$

From this it follows that

$$
R=R_{i}^{i}=-\left(\varrho^{\prime}+3 \varrho^{\prime \prime}\right)
$$


and consequently

$$
R_{i j}-\frac{1}{2} g_{i j} R=\left(\varrho^{\prime \prime}-\varrho^{\prime}\right) u_{i} u_{j}+\frac{1}{2}\left(\varrho^{\prime}+\varrho^{\prime \prime}\right) g_{i j} .
$$

Comparing this with (1.4) and (1.7), we have

$$
\sigma=\frac{1}{k}\left(\varrho^{\prime}-\varrho^{\prime \prime}\right), \quad p=\frac{1}{2 k}\left(\varrho^{\prime}+\varrho^{\prime \prime}\right)
$$

Hence we have the following theorem:

A necessary and sufficient condition that a Riemann space of four dimensions be the space-time continuum of a perfect fluid is that (i) at each point (1.1) is reducible to (1.2) by a real linear transformation of the differentials; (ii) the determinant equation (2.1) admits a simple root and a triple root with simple elementary divisors; (iii) the components $u^{i}$ of the direction determined by the simple root and (3.3) are real and are the components of a velocity vector.

4. World-lines of flow. Einstein chose the left-hand member of equation (1.4) so that the equation should be consistent with the vanishing of the divergence of $T^{i j}$, that is

$$
T^{i j}{ }_{i}=0 \text {, }
$$

where $T^{i j}{ }_{\alpha}$ is a component of the covariant derivative of $T^{i j}$. From (1.7) we have

$$
T^{i j}=\sigma u^{i} u^{j}-p g^{i j}
$$

hence (4.1) gives

$$
\left(\sigma u^{i}\right)_{i} u^{j}+\sigma u^{i} u_{i}^{j}-\frac{\partial p}{\partial x^{i}} g^{i j}=0
$$

From (3.3) it follows that

$$
u_{j} u_{i}^{j}=0
$$

Multiplying (4.3) by $u_{j}$ and summing for $j$, we obtain

$$
\left(\sigma u^{i}\right)_{i}-\frac{\partial p}{\partial x^{i}} u^{i}=0 .
$$

Then (4.3) reduces to

$$
\sigma u^{i} u_{i}^{j}=\left(g^{i j}-u^{i} u^{j}\right) \frac{\partial p}{\partial x^{i}},
$$


or in other form

(4.7) $\sigma\left(\frac{d^{2}}{d s^{2}} x^{j}+\left\{\begin{array}{c}j \\ i k\end{array}\right\} \frac{d x^{i}}{d s} \frac{d x^{k}}{d s}\right)=\left(g^{i j}-u^{i} u^{j}\right) \frac{\partial p}{\partial x^{i}} \quad(i, j, k=1,2,3,4)$,

where $\left\{\begin{array}{c}j \\ i k\end{array}\right\}$ are the Christoffel symbols of the second kind formed with respect to (1.1).

5. When the lines of flow are the curves of parameter $x^{4}$. The congruence of world-lines of flow is defined by the equations

$$
\frac{d x^{1}}{u^{1}}=\frac{d x^{2}}{u^{2}}=\cdots=\frac{d x^{4}}{u^{4}}
$$

If $f^{i}\left(x^{1}, \ldots, x^{4}\right)=a^{i}(i=1,2,3)$, where the $a^{i}$ are arbitrary constants, are independent solutions of (5.1) and we change coorrdinates in accordance with the equations

$$
x^{\prime \alpha}=f^{\alpha}\left(x^{1}, \ldots, x^{4}\right) \quad(\alpha=1,2,3)
$$

in terms of the new coordinates the components of the world-lines of flow are given by

$$
u^{\alpha}=0 \quad(\alpha=1,2,3), \quad u^{4}=\frac{1}{\sqrt{g_{44}}}
$$

In terms of these coordinates equation (4.5) becomes

$$
\frac{\partial}{\partial x^{4}}(\sigma-p)+\sigma \frac{\partial}{\partial x^{4}} \log \sqrt{\frac{-g}{g_{44}}}=0,
$$

and equations (4.6) reduce to

$$
\sigma\left\{\begin{array}{c}
\alpha \\
44
\end{array}\right\}=g_{44} g^{i \alpha} \frac{\partial p}{\partial x^{i}} \quad(\alpha=1,2,3)
$$

$$
\sigma\left(\left\{\begin{array}{c}
4 \\
44
\end{array}\right\}-\frac{\partial}{\partial x^{4}} \log \sqrt{g_{44}}\right)=g_{44} g^{i 4} \frac{\partial p}{\partial x^{i}}-\frac{\partial p}{\partial x^{4}} \quad(i=1,2,3,4) .
$$

From (5.3) it follows that $g_{44}$ must be positive in order that the third condition of the theorem of $\S 3$ be satisfied. Furthermore, in order that 
the curves of parameter $x^{4}$ may be world-lines of flow, it is necessary that at each point there exist a transformation

$$
\begin{array}{ll}
d X^{4}=a_{i}^{4} d x^{i} & (i=1,2,3,4), \\
d X^{\beta}=a_{\alpha}^{\beta} d x^{\alpha} & (\alpha, \beta=1,2,3),
\end{array}
$$

by means of which (1.1) is transformed into (1.2). Substituting the expressions (5.6) in (1.2) and identifying the result with (1.1), we have that the first of (5.6) becomes

$$
d X^{4}=\frac{1}{\sqrt{g_{44}}} g_{i 4} d x^{i} \quad(i=1,2,3,4) .
$$

Then

$$
\begin{aligned}
\left(d X^{4}\right)^{2}-d s^{2} & =\frac{1}{g_{44}}\left(g_{\alpha 4} g_{\beta 4}-g_{44} g_{\alpha \beta}\right) d x^{\alpha} d x^{\beta} \quad(\alpha, \beta=1,2,3) \\
& \equiv A_{\alpha \beta} d x^{\alpha} d x^{\beta}
\end{aligned}
$$

must be a positive definite form. A necessary and sufficient condition is that the determinant $\left|A_{\alpha \beta}\right|$ be positive and that

$$
A_{\alpha \alpha}>0, \quad A_{\alpha \alpha} A_{\beta \beta}-A_{\alpha \beta}^{2}>0 \quad(\alpha, \beta=1,2,3) .
$$

These are readily found to be equivalent to (1.3) and

$$
\left|\begin{array}{ll}
g_{44} & g_{4 \alpha} \\
g_{4 \alpha} & g_{\alpha \alpha}
\end{array}\right|<0, \quad\left|\begin{array}{ll}
g_{44} & g_{4 \alpha} \\
g_{4 \alpha} & g_{\alpha \alpha} \\
g_{\alpha \beta} \\
g_{4 \beta} & g_{\alpha \beta} g_{\beta \beta}
\end{array}\right|>0 \quad(\alpha, \beta=1,2,3)
$$

Since the direction of the line of flow is determined by (2.2) for the simple root $\varrho^{\prime}$ of (2.1), the former becomes for the values (5.3)

$$
R_{4 j}+\varrho^{\prime} g_{4 j}=0 \quad(j=1,2,3,4)
$$

In order that the elementary divisors corresponding to the triple root, $\varrho^{\prime \prime}$, be simple, it is necessary and sufficient that (2.2) for $\varrho^{\prime \prime}$ be satisfied by the values $(1,0,0,0),(0,1,0,0),(0,0,1,0)$ for the covariant components $\lambda_{i}$. Hence if we write (2.2) in the form

$$
\left(R_{j}^{i}+\varrho^{\prime \prime} \delta_{j}^{i}\right) \lambda_{i}=0
$$


the conditions are

$$
R_{j}^{\alpha}+\varrho^{\prime \prime} \delta_{j}^{\alpha}=0 \quad(\alpha=1,2,3 ; j=1,2,3,4)
$$

Combining these results we have the following theorem:

If the functions $g_{i j}$ for a Riemann space of four dimensions satisfy the conditions $g_{44}>0,(1.3),(5.8),(5.9)$ and (5.10), where $\varrho^{\prime}$ and $\varrho^{\prime \prime}$ are different point functions, the space may be interpreted as the space-time continuum of a perfect fluid, the curves of parameter $x^{4}$ being the world-lines of flow.

When $g_{\alpha 4}=0$ for $\alpha=1,2,3$, the inequalities (5.8) become the necessary and sufficient condition that the form $g_{\alpha \beta} d x^{\alpha} d x^{\beta}$ for $\alpha, \beta=1,2,3$ be negative definite. Hence as a corollary we have

When the fundamental quadratic form is reducible to the form

$$
d s^{2}=g_{44} d x^{4}+g_{\alpha \beta} d x^{\alpha} d x^{\beta} \quad(\alpha, \beta=1,2,3)
$$

such that $g_{44}$ is positive and $g_{\alpha \beta} d x^{\alpha} d x^{\beta}$ is negative definite and the conditions

$$
R_{4 \alpha}=0, \quad R_{\alpha \beta}=\lambda g_{\alpha \beta}, \quad R_{44} \neq \lambda g_{44} \quad(\alpha, \beta=1,2,3)
$$

where $\lambda$ is a point function, are satisfied, the space may be interpreted as the space-time continuum of a perfect fluid, the curves of parameter $x^{4}$ being the world-lines of flow.

6. Geodesic lines of flow. From (4.7) it follows that a necessary and sufficient condition that the lines of flow be geodesics is

$$
\left(g^{i j}-u^{i} u^{j}\right) \frac{\partial p}{\partial x^{i}}=0
$$

which condition is satisfied, in particular, when $p$ is constant.

If the coördinates $x^{\alpha}(\alpha=1,2,3)$ are chosen as in $\S 5$, equations $(6.1)$ become

$$
g^{i \alpha} \frac{\partial p}{\partial x^{i}}=0
$$

$$
g^{i 4} \frac{\partial p}{\partial x^{i}}-\frac{1}{g_{44}} \frac{\partial p}{\partial x^{4}}=0 \quad(\alpha=1,2,3 ; i=1,2,3,4)
$$

and from (5.5) we have

$$
\left\{\begin{array}{c}
\alpha \\
44
\end{array}\right\}=0 \quad(\alpha=1,2,3), \quad\left\{\begin{array}{c}
4 \\
44
\end{array}\right\}=\frac{\partial}{\partial x^{4}} \log \sqrt{g_{44}}
$$


If we denote by $[i j, k]$ the Christoffel symbols of the first kind, then from (6.3)

$$
[44,4]=g_{4 i}\left\{\begin{array}{c}
i \\
44
\end{array}\right\}=\frac{1}{2} \frac{\partial g_{44}}{\partial x^{4}}
$$

which is identically satisfied. Proceeding in like manner with $[44, \alpha]$, where $\alpha=1,2,3$, we get

$$
\frac{\partial}{\partial x^{4}}\left(\frac{g_{4 \alpha}}{\sqrt{g_{44}}}\right)=\frac{\partial}{\partial x^{\alpha}} \sqrt{g_{44}} .
$$

If we replace this by

$$
g_{4 \alpha}=\sqrt{g_{44}} \frac{\partial \varphi_{\alpha}}{\partial x^{\alpha}}, \quad V \overline{g_{44}}=\frac{\partial \varphi_{\alpha}}{\partial x^{4}}
$$

where the right-hand member of the first equation is not summed for $\alpha$, it follows from the second of these equations that the functions $\varphi_{\alpha}$ are of the form $\varphi_{\alpha}=\varphi\left(x^{1}, \ldots, x^{4}\right)+F_{\alpha}\left(x_{1}, x_{2}, x_{3}\right)$. If now we take $\varphi$ for $x^{4}$, the linear element assumes the form

$$
d s^{2}=g_{\alpha \beta} d x^{\alpha} d x^{\beta}+2 g_{\alpha 4} d x^{\alpha} d x^{4}+d x^{4^{2}} \quad(\alpha, \beta=1,2,3)
$$

and the functions $g_{\alpha 4}$ are independent of $x_{4}$.

We consider now the case when $p$ is not constant and the linear element is in the form (6.5). From the first three of equations (6.2) we have

$$
\frac{\partial p}{\partial x^{\alpha}}=\lambda g_{\alpha 4} \quad(\alpha=1,2,3), \quad \frac{\partial p}{\partial x^{4}}=\lambda
$$

and the last of (6.2) is satisfied whatever be $\lambda$. From (6.6) we have

$$
\frac{\partial p}{\partial x^{\alpha}}=g_{\alpha 4} \frac{\partial p}{\partial x^{4}}
$$

Since $g_{\alpha 4}$ is independent of $x^{4}$, when we express the condition of integrability of any one of the first three of (6.6) and the last, we obtain

$$
\frac{\partial \lambda}{\partial x^{\alpha}}=g_{\alpha 4} \frac{\partial \lambda}{\partial x^{4}} .
$$


From (6.7) and (6.8) it follows that $\lambda$ is a function of $p$, say $1 / \varphi^{\prime}(p)$, where the prime indicates differentiation with respect to $p$. Then (6.6) become

$$
\frac{\partial \varphi}{\partial x^{\alpha}}=g_{\alpha 4} \quad(\alpha=1,2,3), \quad \frac{\partial \varphi}{\partial x^{4}}=1,
$$

and the linear element is reducible to

$$
d s^{2}=g_{\alpha \beta}^{\prime} d x^{\alpha} d x^{\beta}+\left(d x^{4}\right)^{2} \quad(\alpha, \beta=1,2,3),
$$

where $x^{\prime 4}=\varphi$.

Conversely, when the linear element is in this form equations (6.1) are satisfied by $u^{4}=1, u^{\alpha}=0(\alpha=1,2,3)$, provided that $p$ is a function of $x^{\prime 4}$ alone. From (3.10), (2.1) and (5.12) this means

$$
R_{44}+\frac{R_{\alpha \beta}}{g_{\alpha \beta}}=f\left(x^{\prime 4}\right),
$$

for each $\alpha$ and $\beta$ taking values 1, 2, 3 and not summed. Consequently spaces satisfying (6.11) and the conditions of the last theorem of $\S 5$ with $g_{44}=1$ are the only space-time continua of a perfect fluid for which the lines of flow are geodesics other than the spaces for which $p$ is constant.

7. The geometry of paths for a perfect fluid. Geodesic representation. Equations (4.7) can be written

$$
\frac{d^{2} x^{j}}{d s^{2}}+\Gamma_{i k}^{j} \frac{d x^{i}}{d s} \frac{d x^{k}}{d s}=0 \quad(i, j, k=1, \ldots, 4),
$$

where

$$
\Gamma_{i k}^{j}=\left\{\begin{array}{l}
j\} \\
i k\}
\end{array}\right\}+\frac{1}{2 \sigma}\left(\frac{\partial p}{\partial x^{i}} \delta_{k}^{j}+\frac{\partial p}{\partial x^{k}} \delta_{i}^{j}\right)-\frac{1}{\sigma} g^{j l} \frac{\partial p}{\partial x^{l}} g_{i k},
$$

where $\delta_{i}^{j}=1$ or 0 according as $j$ and $i$ are the same or not.

Professor Veblen and I have based the geometry of a continuum upon equations of the type (7.1), calling their integral curves the paths of the space, and have called the geometry so defined a geometry of paths.* Thus (7.2) defines the functions $\Gamma_{i k}^{j}$ of a geometry of paths for the space of a perfect fluid, the world-lines of flow being paths.

* Cf. various papers in the Proceedings of the National Academy of Sciences, vols. 8 and 9; also a paper by Veblen and Thomas in these Transactions, vol. 25 (1923); and a paper by me in the Annals of Mathematics, ser. 2, vol. 24 (1923), No. 4. 
If $a_{i j}$ is any symmetric covariant tensor of the second order, we have shown that

$$
a_{i j k} \equiv \frac{\partial a_{i j}}{\partial x^{k}}-a_{i l} \Gamma_{j k}^{l}-a_{j l} \Gamma_{i k}^{l} \quad(i, j, k, l=1, \ldots, 4)
$$

are the components of a covariant tensor of the third order; they are generalized covariant derivatives of $a_{i j}$. Moreover, a necessary and sufficient condition that $a_{i j} d x^{i} d x^{j}=$ const. be a first integral of (7.1) is that

$$
a_{i j k}+a_{j k i}+a_{k i j}=0 .
$$

From (7.2) and (7.3) we have

$$
g_{i j k}=\frac{1}{2 \sigma}\left(g_{i k} \frac{\partial p}{\partial x^{j}}+g_{j k} \frac{\partial p}{\partial x^{i}}-2 g_{i j} \frac{\partial p}{\partial x^{k}}\right) .
$$

Since these expressions satisfy (7.4), we have that $g_{i j} d x^{i} d x^{j}=$ const. is a first integral of (7.1). In particular when the constant is zero, we have In the geometry of paths determined by the function (7.2), the world-lines of light in a perfect fluid are paths.

If we put

$$
g_{i j}^{\prime}=e^{2 \varphi} g_{i j},
$$

where $\varphi$ is any point function, then

$$
g^{\prime i j}=e^{-2 \varphi} g^{i j} .
$$

Along any curve we have

$$
d s^{\prime 2}=g_{i j}^{\prime} d x^{i} d x^{j}=e^{2 \varphi} d s^{2}
$$

and (7.1) becomes

$$
\frac{d^{2} x^{j}}{d s^{\prime 2}}+\Gamma_{i k}^{\prime j} \frac{d x^{i}}{d s^{\prime}} \frac{d x^{k}}{d s^{\prime}}=0,
$$

where

$$
\Gamma_{i k}^{\prime j}=\Gamma_{i k}^{j}+\frac{1}{2}\left(\delta_{i}^{j} \frac{\partial \varphi}{\partial x^{k}}+\delta_{k}^{j} \frac{\partial \varphi}{\partial x^{i}}\right) .
$$

If $\left\{\begin{array}{c}j \\ i k\end{array}\right\}^{\prime}$ denote the Christoffel symbols of the second kind formed with respect to (7.8), we have

$$
\left\{\begin{array}{c}
j \\
i k
\end{array}\right\}^{\prime}=\left\{\begin{array}{c}
j \\
i k
\end{array}\right\}+\delta_{i}^{j} \frac{\partial \varphi}{\partial x^{k}}+\delta_{k}^{j} \frac{\partial \varphi}{\partial x^{i}}-g_{i k} g^{j l} \frac{\partial \varphi}{\partial x^{l}} .
$$


Hence from (7.2), (7.10) and (7.11) we have

$$
\begin{array}{r}
\Gamma_{i k}^{\prime j}=\left\{\begin{array}{c}
j \\
i k
\end{array}\right\}^{\prime}-\frac{1}{2}\left(\delta_{i}^{j} \frac{\partial \varphi}{\partial x^{k}}+\delta_{k}^{j} \frac{\partial \varphi}{\partial x^{i}}-2 g_{i k} g^{j l} \frac{\partial \varphi}{\partial x^{l}}\right) \\
+\frac{1}{2 \sigma}\left(\delta_{i}^{j} \frac{\partial p}{\partial x^{k}}+\delta_{k}^{j} \frac{\partial p}{\partial x^{i}}-2 g_{i k} g^{j l} \frac{\partial p}{\partial x^{l}}\right) .
\end{array}
$$

If we make the customary assumption that $\sigma$ and $p$ are functionally related, then a point function $\varphi$ is defined by

$$
\frac{\partial \varphi}{\partial x^{l}}=\frac{1}{\sigma} \frac{\partial p}{\partial x^{l}} \quad(l=1, \ldots, 4) .
$$

When this function is used in (7.6), the equations (7.9) are those of the geodesics of the space with the quadratic form (7.8), as follows from (7.12). Thus we may say either that the paths, and in particular the world-lines of flow, of the given space can be represented by the geodesics in the Riemann space with the quadratic form (7.8), or that by changing the gauge in the given space the paths are geodesics of the space. As a consequence of this result, (6.5), and (7.6) we have the theorem

When the curves of parameter $x^{4}$ are the world-lines of flow of a perfect fuid, the fundamental quadratic form is reducible to

$$
d s^{2}=e^{-2 \varphi}\left(d x^{4^{2}}+g_{4 \alpha} d x^{4} d x^{\alpha}+g_{\alpha \beta} d x^{\alpha} d x^{\beta}\right) \quad(\alpha, \beta=1,2,3)
$$

where $g_{4 \alpha}$ are independent of $x^{4}$, and $\varphi$ is given by (7.13).

Thus in particular for a fluid of constant density, that is, $\sigma=p+a$, when the curves of parameter $x^{4}$ are the lines of flow, we have $g_{44}=c \cdot(p+a)^{2}$, where $a$ and $c$ are constants.

8. The Einstein and de Sitter cosmological solutions. Consider a space whose linear element is of the form

$$
d s^{2}=-e^{\lambda} d r^{2}-r^{2}\left(d \theta^{2}+\sin ^{2} \theta d \varphi^{2}\right)+e^{\nu} d t^{2},
$$

where $\lambda$ and $\nu$ are real functions of $r$ alone; such a space is radially symmetric and static, since $t$ is the coördinate of time. 
In this case we have*

$$
\begin{aligned}
& R_{11}=\frac{1}{2} \nu^{\prime \prime}-\frac{1}{4} \lambda^{\prime} \nu^{\prime}+\frac{1}{4}{\nu^{\prime}}^{2}-\frac{\lambda^{\prime}}{r}, \\
& R_{28}=e^{-\lambda}\left[1+\frac{1}{2} r\left(\nu^{\prime}-\lambda^{\prime}\right)\right]-1, \\
& R_{33}=R_{22} \sin ^{2} \theta \\
& R_{44}=-e^{\nu-\lambda}\left(\frac{1}{2} \nu^{\prime \prime}-\frac{1}{4} \lambda^{\prime} \nu^{\prime}+\frac{1}{4} \nu^{2}+\frac{\nu^{\prime}}{r}\right), \\
& R_{i j}=0
\end{aligned}
$$

The roots of (2.1) are

$$
\varrho_{1}=R_{11} e^{-\lambda}, \quad \varrho_{2}=-e^{-\nu} R_{44}
$$

and

$$
\varrho^{\prime \prime}=\frac{1}{r^{2}} R_{22}=\frac{1}{r^{2}}\left(e^{-\lambda}-1\right)+\frac{1}{2 r} e^{-\lambda}\left(\nu^{\prime}-\lambda^{\prime}\right),
$$

$\varrho^{\prime \prime}$ being a double root. Since one of the roots must be a triple root, we must have either $\varrho_{1}=\varrho^{\prime \prime}$ or $\varrho_{2}=\varrho^{\prime \prime}$. If the latter condition were satisfied, then $\varrho_{1}=\varrho^{\prime}$, and since $R_{i i}+\varrho_{1} g_{i i} \neq 0(i=2,3,4)$, we must have $u^{i}=0$ $(i=2,3,4)$ which is inconsistent with (5.3). Accordingly we must have

$$
\varrho_{1}=\varrho^{\prime \prime}, \quad \varrho_{2}=\varrho^{\prime} .
$$

From (8.2), (8.3) and (8.4) the first of (8.5) gives

$$
\frac{1}{2} \nu^{\prime \prime}-\frac{1}{4} \lambda^{\prime} \nu^{\prime}+\frac{1}{4} \nu^{\prime 2}-\frac{1}{2 r}\left(\lambda^{\prime}+\nu^{\prime}\right)+\frac{1}{r^{2}}\left(e^{\lambda}-1\right)=0
$$

We inquire under what conditions

$$
\varrho^{\prime}=a, \quad \varrho^{\prime \prime}=b \quad(a \neq b),
$$

where $a$ and $b$ are constants. From (8.4) we have

$$
e^{-\lambda}\left(\nu^{\prime}-\lambda^{\prime}\right)=2 r b+\frac{2}{r}\left(1-e^{-\lambda}\right),
$$

* Cf. Eddington, The Mathematical Theory of Relativity, pp. 84, 94. 
and from (8.2), (8.3) and (8.5)

$$
\begin{aligned}
& \frac{1}{2} \nu^{\prime \prime}-\frac{1}{4} \lambda^{\prime} \nu^{\prime}+\frac{1}{4}{\nu^{\prime}}^{2}-\frac{\lambda^{\prime}}{r}=e^{\lambda} b, \\
& \frac{1}{2} \nu^{\prime \prime}-\frac{1}{4} \lambda^{\prime} \nu^{\prime}+\frac{1}{4} \nu^{\prime 2}+\frac{\nu^{\prime}}{r}=e^{\lambda} a .
\end{aligned}
$$

Subtracting these equations we get $e^{-\lambda}\left(\nu^{\prime}+\lambda^{\prime}\right)=r(a-b)$. From this and (8.7) we obtain

$$
\begin{aligned}
& e^{-\lambda} \nu^{\prime}=\frac{r}{2}(a+b)+\frac{1}{r}\left(1-e^{-\lambda}\right), \\
& e^{-\lambda} \lambda^{\prime}=\frac{r}{2}(a-3 b)-\frac{1}{r}\left(1-e^{-\lambda}\right) .
\end{aligned}
$$

Substituting from these equations in the first of (8.8), we get $r(a+b)$ $+2\left(1-e^{-\lambda}\right) / r=0$. From (8.9) it follows that $\nu$ is a constant and from (8.10) that $a=0$. Hence $e^{-\lambda}=1+r^{2} b / 2$. From (3.10) we have that the density $\sigma-p$ is equal to $-3 b / 2 k$. Hence $b$ must be negative. If we put

$$
b=-\frac{2}{R^{2}}, \quad r=R \sin \chi,
$$

the form (8.1) becomes

$$
d s^{2}=-R^{2}\left[d \chi^{2}+\sin ^{2} \chi\left(d \theta^{2}+\sin ^{2} \theta d \varphi^{2}\right)\right]+d t^{2},
$$

which is Einstein's cosmological solution; moreover, $\sigma=-2 p=2 /\left(k R^{2}\right)$. Hence

When a space-time continuum of a perfect fluid admits a linear element of the form (8.1) and both roots are constant, it is Einstein's cosmological space.

When $a$ and $b$ are equal, the principal directions are indeterminate and thus the space cannot be identified with the continuum of a perfect fluid. The solution of the above equations for this case is

$$
e^{-\lambda}=e^{\nu}=1+\frac{a r^{2}}{3}+\frac{c}{r}
$$

where $c$ is a constant. When $a=0$, we have the Schwarzschild solution for empty space, and when $c=0$, de Sitter's cosmological solution.* More-

* Cf. Kottler, Annalen der Physik, vol. 56 (1918), p. 443. 
over, the expressions (8.12) give the most general homogeneous space whose linear element is reducible to (8.1).

9. Radially symmetric space-time continua of a static perfect fluid for which the spaces are of constant Riemann curvature. It is readily shown that when $e^{\lambda}$ in (8.1) is given by

$$
e^{-\lambda}=1-\frac{\alpha r^{2}}{2}
$$

the spaces $t=$ const. are of constant Riemann curvature, which is positive, zero or negative, according as the constant $\alpha$ is positive, zero or negative; and this is the most general form of $e^{\lambda}$ for $t=$ const. to be of this character.

In this case equation (8.6) reduces to

$$
\nu^{\prime \prime}+\frac{1}{2}{\nu^{\prime 2}}^{2}-\frac{\nu^{\prime}}{r\left(1-\frac{\alpha r^{2}}{2}\right)}=0,
$$

and from (8.4), (8.2) and (8.3) we have

$$
\varrho^{\prime \prime}=\frac{\nu^{\prime}}{2 r}\left(1-\frac{\alpha r^{2}}{2}\right)-\alpha
$$

and

$$
\varrho^{\prime}=\frac{1}{2}\left(1-\frac{\alpha r^{2}}{2}\right)\left(\nu^{\prime \prime}+\frac{1}{2}{\nu^{\prime}}^{2}+\frac{\nu^{\prime}}{2 r} \frac{4-3 \alpha r^{2}}{1-\frac{\alpha r^{2}}{2}}\right)
$$

When $\nu^{\prime}=0$, we have the case treated in $\S 8$. If $\nu^{\prime} \neq 0$ and $\alpha \neq 0$, the general integral of (9.2) is

$$
c^{\frac{\nu}{2}}=b \sqrt{1-\frac{\alpha r^{2}}{2}}+c,
$$

where $b$ and $c$ are arbitrary constants. Then from (9.3) and (9.4) we have

$$
\varrho^{\prime}=-\frac{\alpha}{2} \frac{3 b \sqrt{1-\frac{\alpha r^{2}}{2}}}{b \sqrt{1-\frac{\alpha r^{2}}{2}+c}}, \quad \rho^{\prime \prime}=-\frac{\alpha}{2} \frac{3 b \sqrt{1-\frac{\alpha r^{2}}{2}}+2 c}{b \sqrt{1-\frac{\alpha r^{2}}{2}}+c},
$$


and in consequence of (3.10)

$$
\sigma=\frac{\alpha}{k} \frac{c}{b \sqrt{1-\frac{\alpha r^{2}}{2}+c}}, \quad p=-\frac{\alpha}{2 k} \frac{3 b \sqrt{1-\frac{\alpha r^{2}}{2}}+c}{b \sqrt{1-\frac{\alpha r^{2}}{2}}+c} .
$$

From these expressions it follows that $\sigma-p$ is equal to $3 \alpha / 2 k$, and as this cannot be negative, $\alpha$ must be positive. It is readily seen that (9.5) gives Schwarzschild's solution for the gravitational field of a sphere of uniform density.* In fact, if we put $c=-b \sqrt{1-\frac{\alpha r^{2}}{2}}$, we obtain the form of this solution given by Eddington.t

We consider finally the case $\alpha=0$. The general integral of (9.2) is

$$
e^{v}=c^{2}\left(r^{2}+b\right)^{2}
$$

where $b$ and $c$ are arbitrary constants. Then from (9.3) and (9.4) we have

$$
\varrho^{\prime}=\frac{6}{r^{2}+b}, \quad \varrho^{\prime \prime}=\frac{2}{r^{2}+b},
$$

and in consequence of (3.10)

$$
\sigma=p=\frac{4}{r^{2}+b} \text {. }
$$

If $b$ is a positive constant, we have a distribution of matter throughout euclidean space without any point of singularity such that $p$ vanishes at infinity and $\sigma-p$ is zero everywhere, meaning a uniform distribution of a finite amount of matter throughout the space. $\neq$

*Sitzungsberichte der Preubischen Akademie der Wissenschaften, 1916, p. 424.

† Loc. cit., p. 169.

$\ddagger$ Compare these results with the remark at the close of $\S 7$.

Princeton Univeraity,

Princeton, N.J. 